Research Paper

\title{
Chlorpheniramine attenuates histamine-mediated aquaporin 5 downregulation in human nasal epithelial cells via suppression of NF-KB activation
}

\author{
Yung-Lung Chang1, Chun-Shu Lin², Hsing-Won Wang ${ }^{3,4}$, Kai Ren Jian ${ }^{1}$, Shao-Cheng Liu ${ }^{3 凶}$ \\ 1. Department of Biochemistry, National Defense Medical Center \\ 2. Department of Radiation Oncology, Tri-Service General Hospital, National Defense Medical Center \\ 3. Department of Otolaryngology-Head and Neck Surgery, Tri-Service General Hospital, National Defense Medical Center \\ 4. Department of Otolaryngology-Head and Neck Surgery, Shuang Ho Hospital Taipei, Taiwan, Republic of China
}

$\triangle$ Corresponding author: Shao-Cheng Liu, M.D. PhD., Assistant Professor, Department of Otolaryngology-Head and Neck Surgery, Tri-Service General Hospital, National Defense Medical Center, No. 325, Sec. 2, Cheng-Gong Road, Neihu District, Taipei, Taiwan 114, R.O.C. Tel: 886-2-8792-7192; Fax: 886-2-8792-7193; E-mail: m871435@ndmctsgh.edu.tw

(C) Ivyspring International Publisher. This is an open access article distributed under the terms of the Creative Commons Attribution (CC BY-NC) license (https://creativecommons.org/licenses/by-nc/4.0/). See http://ivyspring.com/terms for full terms and conditions.

Received: 2017.06.22; Accepted: 2017.09.01; Published: 2017.09.30

\begin{abstract}
Background: Aquaporin 5 (AQP5) is most likely the primary water channel in the human nasal mucosa and acts as a key tight junction protein. The signaling cascades responsible for AQP5 regulation are still works in progress.

Objective: This study sought to determine the effects of histamine and chlorpheniramine on AQP5 expression in human nasal epithelial cells $(\mathrm{HNEPC})$ and to detect the signaling cascades responsible for these effects.

Methods: HNEpC were cultured with four concentrations of histamine or chlorpheniramine in vitro. The sub-cellular distribution of AQP5 was explored using immunocytochemistry. The pharmacologic effects of histamine and chlorpheniramine on the expression of the phosphorylation of cyclic adenosine monophosphate-responsive element binding protein (P-CREB), the AQP5 and the NF-KB protein were examined using Western blotting.

Results: AQP5 was found to be located in cell membrane and cytoplasm and present in every group without significant difference. Histamine inhibits the expression of AQP5 and P-CREB in HNEpC, while chlorpheniramine dose-dependently increases these protein levels with statistical significance. HNEpC treated with histamine and chlorpheniramine in turn showed the same trends as those intervened separately with these two drugs. Moreover, chlorpheniramine had the ability to reverse the inhibitory effect of histamine. Western blotting analysis revealed that after incubation with 10-4 M histamine, NF-kB protein was significantly heightened by $165 \%$ compared with the untreated control group. Again, such increase can be significantly reversed after chlorpheniramine treatment.

Conclusions: The current study demonstrated that histamine inhibits CREB phosphorylation in HNEpC, which results in decreased AQP5 expression via activation of NF-KB pathway. Chlorpheniramine attenuates the inhibitory effect of histamine in $\mathrm{P}-\mathrm{CREB} / \mathrm{AQP5}$ expression via suppression of NF- $\mathrm{kB}$ signal cascades. This observation could provide additional insight into the anti-inflammatory effects of $\mathrm{HI}$-antihistamines that contribute to maintain airway surface liquid and mucosal defense.
\end{abstract}

Key words: AQP5, cAMP, CREB, antihistamine, NF-KB, nasal mucosa, histamine

\section{Introduction}

Both allergic rhinitis (AR) and chronic rhinosinusitis (CRS) are chronic inflammatory diseases of the upper airways that have a major impact on the quality of life of patients. The treatment is straightforward, and most patients response positively to anti-histamines and steroid nasal spray. However, the etiology and pathogenesis of CRS remain largely unknown. Some patients with CRS 
will progress to recurrent or persistent polyposis and show poor treatment response, while some do not. ${ }^{1}$ Among hypotheses proposed to explain all or part of the clinical CRS spectrum, the "immune barrier hypothesis" posits that an intact barrier with tight epithelial junctions is necessary for a healthy nasal mucosa. Defects in this protective mechanical and innate immune barrier may lead to an abnormal microbiome, probably in biofilm format. ${ }^{2}$ Impaired mucociliary flow and a porous mechanical barrier caused increased access of pathogen-associated molecular patterns, thus stimulating a compensatory response and a subsequent dysregulated inflammatory cascade.

Fluid and macromolecule secretion by submucosal glands in mammalian airways is believed to play an important role in airway antimicrobial defense and surface liquid homeostasis. ${ }^{3}$ Aquaporins (AQPs) are integral membrane proteins that function as molecular water channels in a variety of fluid-transporting tissues.4,5 AQPs facilitate water transport across epithelia and play an important role in normal nasal physiology. The deletion or decreased expression of AQPs dysregulates the tight junction function and permeability of the barrier, and is often associated with disease.6,7 AQPs 1-5 are all expressed in the human nasal mucosa; however only AQP5 is found in the apical membrane of columnar cells of the superficial epithelium and submucosal gland acinar cells. ${ }^{7,8}$ According to Kreda et al. ${ }^{5}$, AQP5 is most likely the primary water channel in the human nasal mucosa and acts as a key tight junction protein in the maintenance of mucosal water homeostasis. Abnormal AQP5 expressions with viscous gland secretions were known to promote bacterial adhesion and inhibit bacterial clearance by impeding ciliary function and were thought to be one of the pathogeneses of cystic fibrosis. ${ }^{9}$ In recent years, the association between the pathophysiology of CRS and AQP5 has been partly studied7, but the signaling cascades responsible for these effects are still works in progress.

Histamine is the most important preformed mediator in AR and CRS. Histamine causes mucus secretion, vasodilatation, tissue edema, and sneezing. However, little is known about the direct involvement of histamine in airway epithelial AQP5 regulation. Previous research has demonstrated that histamine downregulates AQP5 expression via inhibiting cyclic adenosine monophosphate (cAMP) response element-binding protein (CREB) phosphorylation $(p-C R E B){ }^{10}$, but the mechanism involved has not been clearly defined to date. H1-antihistamines work primarily through membrane-associated G protein-coupled receptors and blockage of downstream signaling cascades. ${ }^{11}$ Cumulative clinical evidence indicated additional anti-inflammatory effects of H1-antihistamines. However, its implication in pathophysiology of CRS, especially in patients with polyps was largely unknown. To further understand the pathophysiological role of histamine, H1-antihistamine and AQP5 in regulating human nasal secretion, primary cultures of human nasal epithelial cells (HNEpC) were established. This study hypothesized that histamine downregulates $\mathrm{p}$-CREB and AQP5 via activation of NF-kB pathway and H1-antihistamine can attenuate it. Description of this pathway could provide theoretical basis for studying disorders of water metabolism; and the results thus obtained may shed light on novel approaches to treating inflammatory airway disease with hyperviscous gland secretions.

\section{Materials and Methods}

\section{Primary cultures of HNEpC and grouping}

All cell culture reagents were purchased from Sigma-Aldrich (St. Louis, MO) unless otherwise stated. Nasal mucosa specimens were obtained after partial inferior turbinectomy performed on 8 patients (5 men and 3 women) who suffered from chronic nasal congestion due to turbinate hypertrophy or allergic rhinitis. The establishment of the experimental model employed had been published in our previous report. ${ }^{12}$ In brief, HNEpC primary cultures were obtained using the dispase dissociation technique and the epithelia cells were resuspended in keratinocytes serum-free medium and cultured in a $25-\mathrm{ml}$ flask in a humidified chamber with $95 \%$ air and $5 \% \mathrm{CO}_{2}$ at $37^{\circ} \mathrm{C}$. Histamine and chlorpheniramine, a first-generation H1-antihistamine, were used as testing agents. There were four groups defined by the concentration of testing agents: $0,10^{-6} \mathrm{M}, 10^{-5} \mathrm{M}$, and the $10^{-4} \mathrm{M}$. When the cells reached $80 \%$ confluence, they were induced by histamine or chlorpheniramine for 4 or 24 hours, respectively. Finally, three HNEpC groups were first stimulated with $10^{-4} \mathrm{M}$ histamine for 4 hours, followed by 24 -hour exposure to $0,10^{-5} \mathrm{M}$, and $10^{-4} \mathrm{M}$ chlorpheniramine, respectively. The untreated cells (no histamine or chlorpheniramine) were used as the control group.

\section{Immunocytochemistry}

Cells from primary cultures were passaged into immunocytochemical chamber slides on passage 1 and were trypsinized after treatment with the above protocol. The cell suspension was prepared in PBS and cytocentrifugation was performed at $1500 \mathrm{rpm}$ for 5 minutes, to allow the cells to bind to the glass slide. The cytospin slides were rinsed three times with PBS and immunostained (ABC immunoperoxidase) with 
the primary antibody (goat-anti-AQP5, Santa Cruz Biotechnology Inc, No.sc-9890, 1:250), as described previously. The sections were incubated with 3-amino-9-ethylcarbazole (AEC) and AQP5 was visualized with a Nikon Eclipse E400 microscope. The specificity of the antibody was ensured by omitting the primary antibody in control sections.

\section{Assay of AQP5, p-CREB and NF-KB levels}

Nuclear and membranous protein was isolated separately for the detection of membranous AQP5, nuclear p-CREB (Ser133) and nuclear NF-kB using the Nuclear-Cytosol Extraction Reagent Kit (Pierce, Rockford, IL), with the loading control: HSP-90, SP-1 and GAPDH, respectively. Samples were subjected to $12 \%$ SDS-PAGE and were electroblotted onto polyvinylidene difluoride membranes (Millipore Corporation, Billerica, MA). The membranes were blocked and then incubated sequentially with both primary (goat-anti-AQP5 antibody at 1:200 dilutions, rabbit-anti-p-CREB (Ser133) antibody at 1:250 dilutions, anti-NF-kB p65 antibody, $0.5 \mu \mathrm{g} / \mathrm{ml}$ ) and secondary antibodies. The relative band intensity was analyzed using TotalLab Quant software (TotalLab

Ltd., Newcastle, UK) and was expressed as ratio of protein to control.

\section{Statistical Analysis}

SPSS 13.0 software (SPSS Inc, Chicago, IL) was used for statistical analysis. Statistical significance was evaluated using analysis of variance, followed by a Dunnet test. A $p$ value $<0.05$ was considered to indicate statistical significance. Each data point was expressed as the mean (SD).

\section{Ethical considerations}

The research protocol (NO: 1-104-05-143) has been reviewed and approved by the Institutional Review Board of Tri-Service General Hospital.

\section{Results}

\section{Immunocytochemistry}

AQP5 was present in every group (Fig. 1). Images at high magnification showed subcellular localization of AQP5, obviously in cell membrane and cytoplasm. There were trends of higher AQP5 expression after chlorpheniramine treatment but lower AQP5 expression after histamine stimulation,

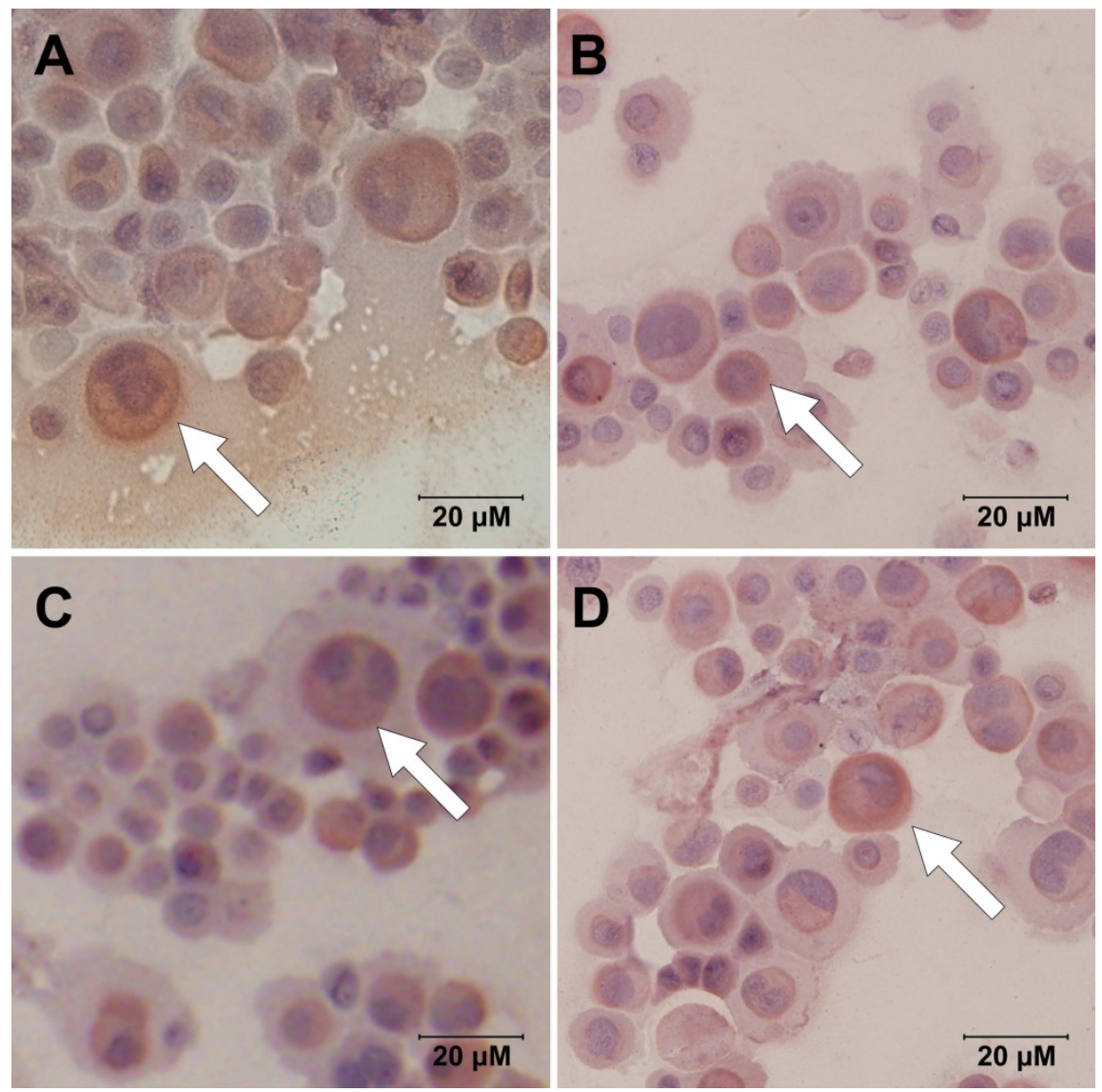

Figure 1. Immunocytochemistry images demonstrate expressions of AQP5 within HNEpC at different interventions: (A) control, (B) 10-4M histamine, (C) 10-4M chlorpheniramine, and (D) 10-4M histamine and chlorpheniramine (scale bars $=20 \mu \mathrm{m}$ ). indicating stimulatory effect of chlorpheniramine and inhibitory effect of histamine, respectively. However, no apparent difference was noted between each group after intervention.

\section{Western blotting}

The protein level of nuclear p-CREB and membranous AQP5 detected by Western blotting after histamine stimulation are shown in Fig. 2. As can be seen, both levels decrease in a concentration-dependent manner. Incubation with $10^{-6} \mathrm{M}$, $10^{-5} \mathrm{M}$, and $10^{-4} \mathrm{M}$ of histamine led to decreases in AQP5 of $1.66 \%, 36.05 \%$, and $60.36 \%$, respectively compared with the $0 \mathrm{M}$ group. p-CREB protein decreased significantly by $3.65 \%, 20.23 \%$, and $24.52 \%$ in the $10^{-6} \mathrm{M}, 10^{-5} \mathrm{M}$, and $10^{-4} \mathrm{M}$ groups, respectively compared with the $0 \mathrm{M}$ group. Both AQP5 and $p$-CREB expression in $10^{-5}$ and $10^{-4} \mathrm{M}$ groups showed statistically significant differ- 
ences after histamine intervention, compared with those of the untreated control group $(p<0.05)$.
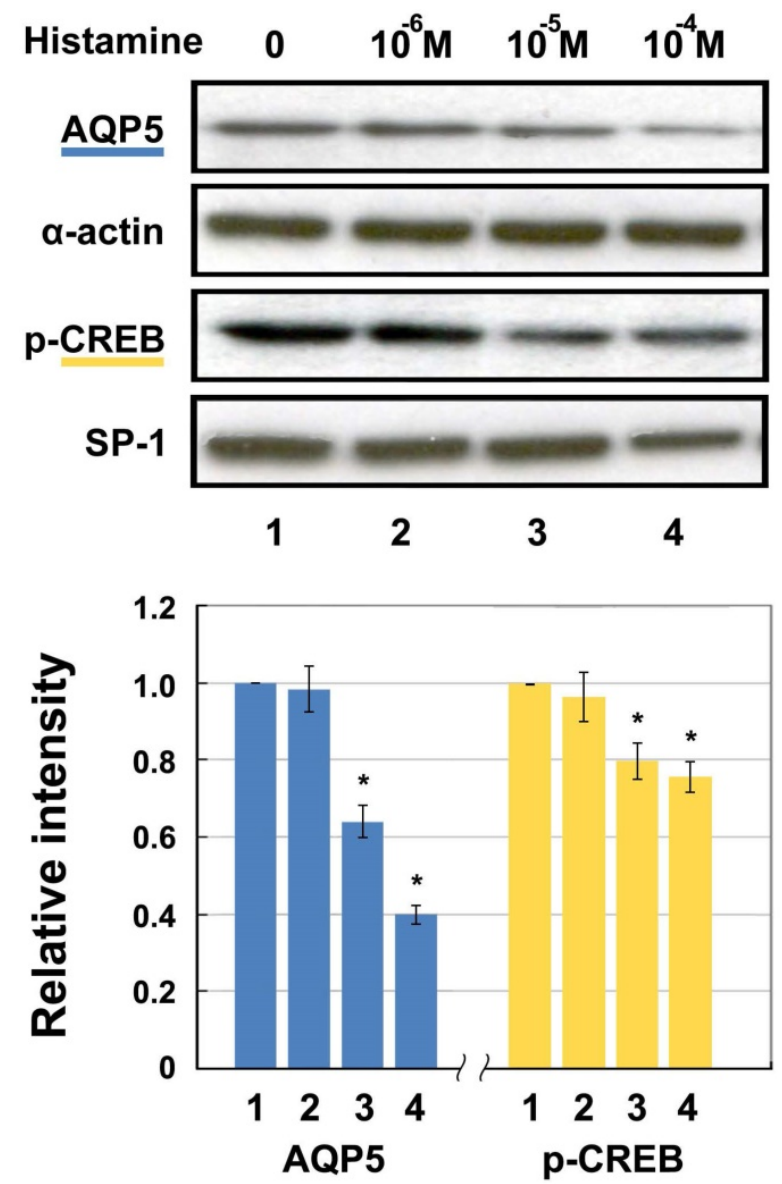

Figure 2. Western blotting for AQP5 and P-CREB (Ser133) protein levels in HNEpC incubated with histamine. $\alpha$-actin and SP-1 were used as loading control for AQP5 and P-CREB, respectively. Data represent the fold increase in expression of target protein relative to control. ${ }^{*} \mathrm{p}<.05$ versus untreated control.

A completely opposite result was shown after intervention with chlorpheniramine (Fig. 3). The expression of AQP5 showed dose-dependent relationships with $10^{-6} \mathrm{M}, 10^{-5} \mathrm{M}$, and $10^{-4} \mathrm{M}$ of chlorpheniramine, leading to increases of $3.84 \%$, $7.62 \%$, and $18.26 \%$, respectively compared with the 0 $\mathrm{M}$ group. Except for the $10^{-6} \mathrm{M}$ group, all differences were statistically significant $(p<0.05)$. $p$-CREB protein in the $10^{-6} \mathrm{M}, 10^{-5} \mathrm{M}$, and $10^{-4} \mathrm{M}$ groups was significantly increased by $6.65 \%, 38.53 \%$, and $42.85 \%$, respectively compared with the $0 \mathrm{M}$ group $(\mathrm{p}<0.05)$.

Experimental results of HNEpCs incubated first with $10^{-4} \mathrm{M}$ histamine for 4 hours and then with 0 $\mathrm{M}, 10^{-5} \mathrm{M}$, and $10^{-4} \mathrm{M}$ chlorpheniramine for 24 hours showed the same trends as those intervened with test agents separately. Histamine obviously inhibits the expression of AQP5 and p-CREB, while chlorpheniramine increases these protein levels dose-dependently (Fig. 4). Incubation with $10^{-4} \mathrm{M}$ histamine led to decreases of $56.95 \%$ and $25.22 \%$ in AQP5 and p-CREB expression, respectively, as compared with the untreated control group (no histamine or chlorpheniramine) $(\mathrm{p}<0.05)$. Chlorpheniramine has the ability to reverse the inhibitory effect of histamine and the decrease in AQP5 and p-CREB expression were $54.11 \%$ and $11.27 \%$ at $10^{-5} \mathrm{M}$ and $4.29 \%$ and $3.87 \%$ at $10^{-4} \mathrm{M}$, respectively, as compared with the untreated control group.

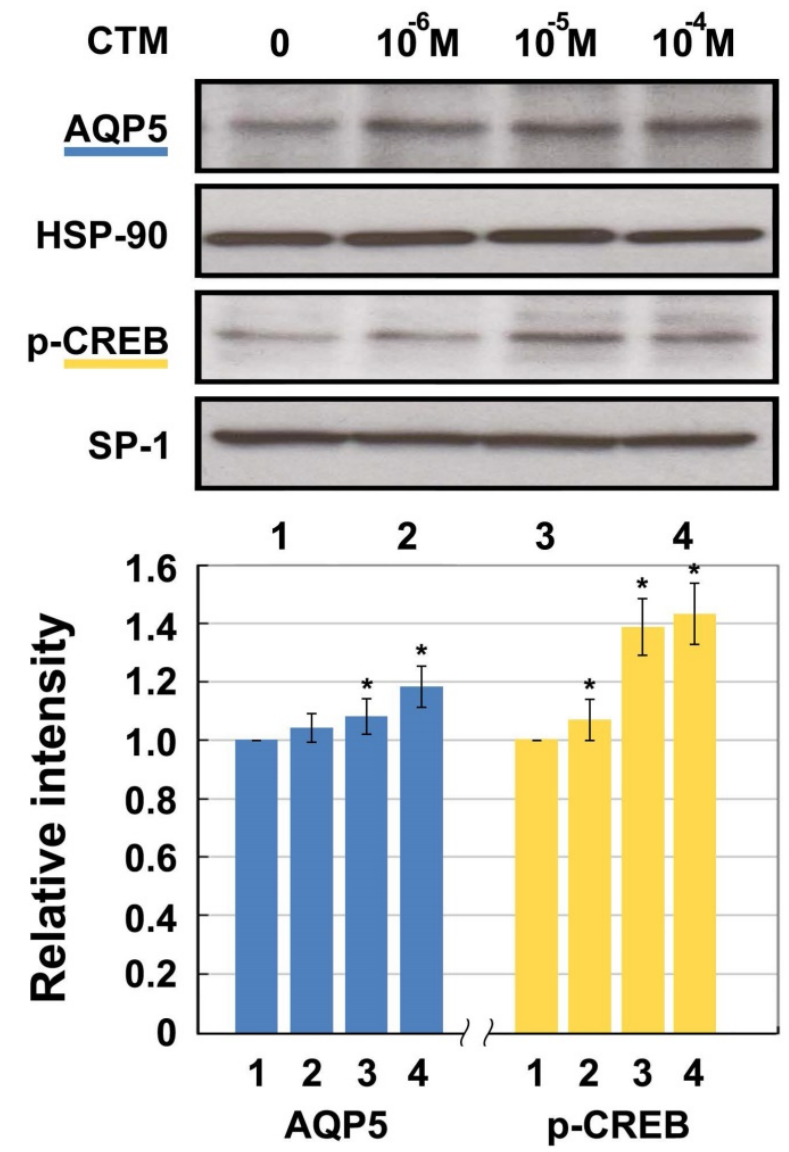

Figure 3. Western blotting for AQP5 and p-CREB (Ser133) protein levels in HNEpC incubated with chlorpheniramine (CTM). HSP-90 and SP-1 were used as loading control for AQP5 and P-CREB, respectively. Data represent the fold increase in expression of target protein relative to control. ${ }^{*} \mathrm{p}<.05$ versus untreated control.

Moreover, NF-kB protein assayed by Western blotting after incubation with $10^{-4} \mathrm{M}$ histamine was significantly heightened by $165.0 \% \quad(p<0.05)$ compared with the untreated control group. Again, these increases can be markedly reversed after chlorpheniramine treatment and the decreases in NF-KB were $20.37 \%$ and $23.45 \%$ at $10^{-5} \mathrm{M}$ and $10^{-4} \mathrm{M}$, respectively, as compared with those without chlorpheniramine treatment (Fig. 4, Group 4) $(\mathrm{p}<$ 0.05). 


\section{Discussion}

Water transport in nasal mucosa play a very important role in the pathogenesis of $A R$ and CRS. AQP5 is a water-selective transporting protein and is generally expressed in the membrane and cytoplasm of the secretory cells in glandular tissues., ${ }^{4,7}$ It is proposed that AQP5 is more important than other AQPs in a nasal mucosa and is a rate-limiting barrier in mucin production. Under hypo-osmotic stress, AQP5 is obviously downregulated and this repression might promote clogging or increase the risk of infections with pathogenic bacteria. ${ }^{13}$ Mice lacking AQP5 show increased bronchoconstriction of the airways. ${ }^{14,15}$ However, Seno et al. ${ }^{8}$ noted no difference in expression of AQP5 in the human nasal mucosa and its mRNA among AR, CRS and control patients. Shikani et al. ${ }^{7}$ showed that the epithelial expression of
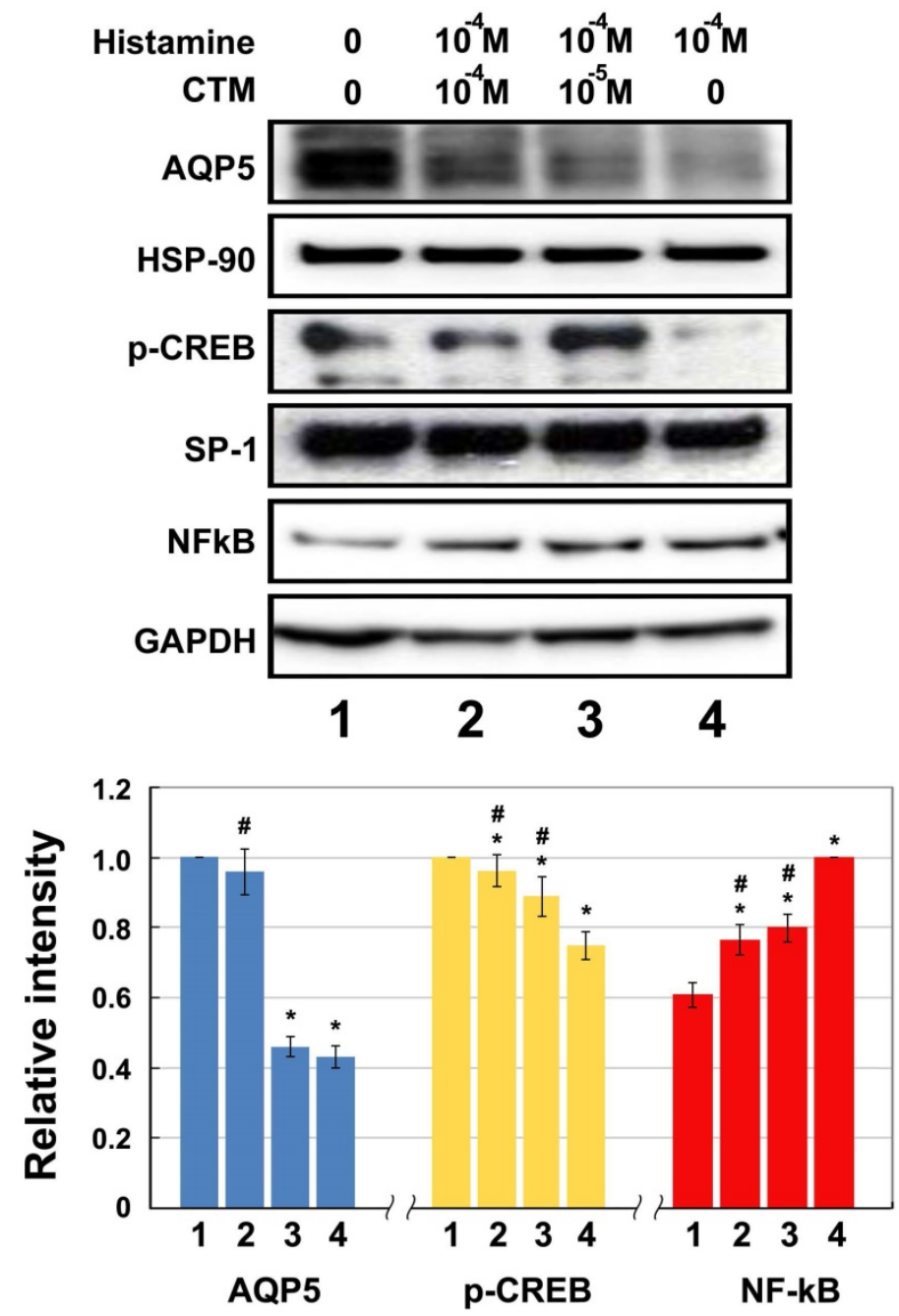

Figure 4. Western blotting for relative expression of p-CREB, AOP5 and NF-kB elicited by histamine and effect of chlorpheniramine (CTM) $(N=8)$. Data represent the fold increase in expression of target protein. ${ }^{*} p .05$ versus untreated control (group 1); \# $p<.05$ versus histamine-treated cells (group 4). CTM attenuated significantly the histamine effect and the expression of NF-KB was significantly lowered by $26.31 \%$ and $31.38 \%$ at $10^{-5}$ and $10^{-4} \mathrm{M}$, respectively, as compared with those without CTM treatment (group 4).
AQP5 was significantly lower in patients with nasal polyps as compared with the control. Whether the compromise of mucosal epithelial barrier is caused by loss or internalization of epithelial AQP5 remains unclear. Therefore, the role of AQP5 in pathogenesis of $\mathrm{AR}$ and CRS remained unclear and the lack of literature in this field prompted our in vitro research.

Previous studies have suggested that the cAMP-protein kinase A (PKA)/p-CREB pathway is involved in AQP5 expression. ${ }^{16,17}$ Research on lung epithelial cell lines in mice revealed that the agonist of cAMP can increase the expression of $\mathrm{p}$-CREB and AQP5 while these effects can be blocked by a PKA inhibitor. ${ }^{16-20}$ CREB is a well-known transcription factor and CREB phosphorylation at serine 133 is reported to be critical in AQP5 expression. ${ }^{16-18}$ As a homodimer or heterodimer, $\mathrm{p}$-CREB binds to the conserved CREB-responsive elements present at the promoter region of the AQP5 gene, and the gene transcriptions of AQP5 are induced. ${ }^{17}$ The current results are consistent with the findings that $\mathrm{p}$-CREB has a major role in AQP5 expression of HNEpC and that higher expression of p-CREB was associated with increased AQP5 protein. Therefore, to understand the regulation of AQP5, the implications of CREB phosphyrylation merit more in-depth study.

Histamine plays a major role in AR or CRS and causes the hypersecretion of nasal mucosa ${ }^{11}$; hence, it is important to delineate the regulatory mechanism of histamine signal cascades. CREB is an important mediator of the effect of histamine..$^{21}$ A study showed that CREB plays an important role in regulating histamine action at the gene expression level, and its phosphorylation following histamine stimulation has been reported. Hegyi et al. ${ }^{21}$ used a mouse model that lacked endogenous histamine to study how histamine deficiency impacts CREB signaling. They found that cells show higher constitutive CREB activity in the absence of histamine and intracellular cAMP levels increased greatly. In their study on human nasal epithelial cells, Wang et al. ${ }^{10}$ reported that histamine downregulated p-CREB which led to repression of AQP5 mRNA and protein in a concentration-dependent fashion, indicating a role for histamine-mediated CREB function. These data are consistent 
with the current findings that dose-dependent decreases of p-CREB and AQP5 were observed after 4-hour exposure to histamine. The same experiment was repeated using BEAS-2B cells (ATCC® CRL-9609TM), a cell line derived from normal human bronchial epithelial cells, and consistent results were obtained (data not shown). Hence, the histamine-medicated $\mathrm{p}$-CREB and AQP5 repression is a common pathway in airway epithelial cells.

H1-antihistamines are the most commonly prescribed medication for patients with AR; however, its efficacy for CRS with polyps is unclear. Studies showed that although oral H1-antihistamine was effective in reducing sneezing and rhinorrhea, polyps remained unchanged. ${ }^{22}$ Previous studies highlighted additional anti-inflammatory effects of H1-antihistamines. Indeed, these drugs not only antagonize an immediate allergic reaction, but also exert inhibitory effects on the expression of adhesion molecules, and on the release of leukotrienes, inflammatory cytokines or chemokines that result in inhibitions of eosinophil migration and neutrophil infiltration during the late-phase reaction. ${ }^{22,23}$ Our previous research reported the anti-mitogenic property of H1-antihistamines in HNEpC cultures ${ }^{12}$ that is beneficial in treatment against airway remodeling. In this study, another anti-inflammatory effect of H1-antihistamines in CREB phosphorylation was demonstrated for the first time. In our model, cells maintained higher constitutive p-CREB activity in the presence of chlorpheniramine and increased greatly membranous AQP5 expression in a concentration-dependent fashion. These observations suggest that treatment with chlorpheniramine is promising to CRS patients because increased expression of AQP5 contributes to maintain an isotonic airway surface liquid and mucosal defense. Our previous study also revealed suppression of H1, muscarinic- 1 and 3 receptors after treatment with H1-antishitamines but the relationship between these changes and $\mathrm{p}$-CREB/AQP5 requires further exploration. Some investigators considered the additional anti-inflammatory properties of H1-antihistamine unrelated to H1-receptor antagonism. ${ }^{24}$ However, other studies are in favor of an H1-receptor-mediated mechanism. The implication of the H1-receptor in the additional effects of H1-antihistamines thus remains controversial. As known, chlorpheniramine is not a specific H1-receptor antagonist and its lack of receptor selectivity and antimuscarinic features might all contribute to the downregulation of $\mathrm{p}$-CREB and AQP5. Future studies using BEAS-2B cells to qualify the specific receptor-mediated mechanism in knockout or knockdown H1, M1 or M3 receptor might be useful.

According to the literature and the present results, AQP5 is expressed both in the membrane and cytoplasm, regulated by kinases and exhibits a pattern of endocytosis that affects tight junction regulation.20,25 It has been recognized that hormones such as vasopressin, estrogen and hormone peptide YY regulate the expression and intracellular trafficking of certain AQPs. ${ }^{26}$ Parvin et al. demonstrated that AQP5 trafficked from the intracellular vesicles to the apical membrane in response to stimulation of muscarinic receptors or to an increase in the intracellular level of calcium ions in human salivary gland cells. ${ }^{27}$ All these reports imply redistribution of AQP5 from inside the cells to the apical membrane on stimulation with secretagogues. The current results showed that the expression of AQP5 after histamine or chlorpheniramine treatment is only affected in membranous extraction, but not in whole cell lysate (data not shown). It is speculated that the loss of epithelial AQP5 after intervention is due to endocytosis or downregulation, suggesting that it is the actual regulation of AQP5 expression that matters, not just the total levels of the protein in the tissue. The findings also demonstrated that AQP5 is internalized in HNEpC after 4-hour histamine exposure while this effect can be attenuated by chlorpheniramine. This translocation of AQP5 in HNEpC due to $\mathrm{Ca}^{2+}$ signaling is a potential mechanism as in salivary gland. In their study using human salivary gland cells ${ }^{28}$, Kim et al. reported that histamine increased intracellular $\mathrm{Ca}^{2+}$ concentration that can be inhibited by chlorpheniramine. However, they showed that histamine resulted in translocation of AQP5 to the plasma membrane and this finding is different from that obtained in this study and by Wang et al. ${ }^{10} \mathrm{~A}$ possible reason for such discrepancy is that those effects may be tissue-specific, which needs further clarification. However, the translocation of AQP5 from the granule membrane to the apical membrane on exocytosis provoked by chlorpheniramine is still plausible because $\mathrm{H} 1$ receptors are related to $\mathrm{Ca}^{2+}$ mobilization. According to the current results, H1-antihistamines might contribute to retain AQP5 in a vesicle-recycling compartment, thus escaping the degradative pathway.

NF- $\mathrm{KB}$ is an inducible cellular transcription factor with polytranscriptional regulatory capacities. It plays a vital role in the regulation of AR and CRS cytokine networks. After the NF-kB pathway was activated, the gene transcription of an array of pro-inflammatory mediators and inflammatory cytokines, including IL-1b and TNF- $\alpha$, is induced. ${ }^{29}$ It has been reported that NF-kB pathway 
downregulates the expression of AQP5 in AR rats, by inhibiting p-CREB. Wang et al. ${ }^{19}$ showed that this signal cascade works through increased transcription of IL-1b while Skowron-zwarg et al..$^{30}$ demonstrated that TNF-a was involved and suppressed cAMP-response element-dependent gene expression by competitive binding to CREB-binding protein. ${ }^{19}$ The inhibition of biosynthesis of $\mathrm{p}$-CREB and AQP5 through NF-kB pathway is consisted with the current results obtained using human specimens. Histamine can activate the transcription factors NF-kB through H1-receptor in the human alveolar epithelial cell line A549 derived from adenocarcinoma. ${ }^{31}$ Hence, characterizing how H1-antihistamines implicate the NF-kB pathway in HNEpC has aroused research interest. Previous studies with H1-antihistamines have reported that they can diminish DNA binding of NF-kB. ${ }^{22}$ Furthermore, H1-antihistamines can behave as inverse agonists ${ }^{11}$, inhibiting H1-receptor constitutive activation of NF-kB. ${ }^{23}$ Roumestan et al. ${ }^{24}$ provided evidence that H1-antihistamines downregulate NF-kB activities through H1-receptor-dependent and -independent pathways, such as direct inhibition of arachidonate 5 -lipoxygenase. The current findings were in line with previous results that using chlorpheniramine represses NF-kB and attenuates its inhibitory effect in CREB phosphorylation and AQP5 internalization. Although this effect might also be H1-receptor-independent because chlorpheniramine is not a specific H1-receptor antagonist, this study still offers a molecular basis for H1-antihistamines in treating CRS and yield useful insights into the physiological properties of airway tissue with abundant AQP5 expression. In-depth investigation using more specific H1-receptor antagonist and cell line such as BEAS-2B is needed to further elucidate its implication in AQPs regulatory pathway and to study the water metabolism disorders of AR and CRS.

\section{Conclusion}

The current study demonstrated that histamine inhibits CREB phosphorylation in HNEpC, which results in decreased AQP5 expression via activation of NF-kB pathway. Chlorpheniramine attenuates the inhibitory effect of histamine in p-CREB/AQP5 expression via suppression of NF-kB signal cascades. This observation could provide additional insight into the anti-inflammatory effects of H1-antihistamines that contribute to maintain airway surface liquid and mucosal defense. However, how H1-antihistamines downregulate NF-kB activities remain unclear. Whether H1-receptor-dependent or -independent pathway is involved in the mechanism merits further exploration.

\section{Acknowledgements}

The study was partially supported by a grant from the Tri-Service General Hospital, National Defense Medical Center MAB-106-004.

\section{Author contribution:}

Yung-Lung Chang, PhD, ylc7305@gmail.com: study design, data collection, article and images review/editing.

Kai Ren Jian, k199311192005@gmail.com: western blot analysis, data collection.

Chun-Shu Lin, MD, chunshulin@gmail.com: article and images review/editing.
Hsing-Won
Wang,
$\mathrm{MD}$,
$\mathrm{PhD}$, w0512n@ms15.hinet.net: article and images review/editing.

Shao-Cheng

Liu,

MD,

$\mathrm{PhD}$, m871435@ndmctsgh.edu.tw: study design, data collection, immunohistochemical staining, literature search, images editing, article drafting, article submission.

\section{Competing Interests}

The authors have declared that no competing interest exists.

\section{References}

1. Veloso-Teles R, Cerejeira R. Endoscopic sinus surgery for chronic rhinosinusitis with nasal polyps: Clinical outcome and predictive factors of recurrence. Am J Rhinol Allergy. 2017 Jan 1;31(1):56-62.

2. Kern RC, Conley DB, Walsh W, et al. Perspectives on the etiology of chronic rhinosinusitis: an immune barrier hypothesis. Am J Rhinol 2008;22(6):549-59.

3. Borok Z, Verkman AS. Lung edema clearance: 20 years of progress: invited review: role of aquaporin water channels in fluid transport in lung and airways. J Appl Physiol (1985). 2002 Dec;93(6):2199-206.

4. Song Y, Verkman AS. Aquaporin-5 dependent fluid secretion in airway submucosal glands. J Biol Chem. 2001 Nov 2;276(44):41288-92.

5. Kreda SM, Gynn MC, Fenstermacher DA, Boucher RC, Gabriel SE. Expression and localization of epithelial aquaporins in the adult human lung. Am J Respir Cell Mol Biol. 2001 Mar;24(3):224-34.

6. Shen Y, Wang Y, Chen Z, Wang D, Wang X, Jin M, Bai C. Role of aquaporin 5 in antigen-induced airway inflammation and mucous hyperproduction in mice. J Cell Mol Med. 2011 Jun;15(6):1355-63.

7. Shikani AH, Sidhaye VK, Basaraba RJ, Shikani HJ, Alqudah MA, Kirk N, Cope E, Leid JG. Mucosal expression of aquaporin 5 and epithelial barrier proteins in chronic rhinosinusitis with and without nasal polyps. Am J Otolaryngol. 2014;35(3):377-83.

8. Seno S, Ogawa T, Shibayama M, Kouzaki H, Shimizu T. Expression and localization of aquaporin 1, 2, 3, 4, and 5 in human nasal mucosa. Am J Rhinol Allergy May-Jun 2012;26(3):167-71.

9. Kitchen P, Öberg F, Sjöhamn J, Hedfalk K, Bill RM, Conner AC, Conner MT, Törnroth-Horsefield S. Plasma Membrane Abundance of Human Aquaporin 5 Is Dynamically Regulated by Multiple Pathways. PLoS One. 2015 Nov 16;10(11):e0143027.

10. Wang W, Wang X, Ma L, Zhang R. Histamine downregulates aquaporin 5 in human nasal epithelial cells. Am J Rhinol Allergy. 2015;29(3):188-92.

11. Simons FE. Advances in H1-antihistamines. N Engl J Med 2004;351:2203-2217.

12. Liu SC, Lin CS, Chen SG, Chu YH, Lee FP, Lu HH, Wang HW. Effect of budesonide and azelastine on histamine signaling regulation in human nasal epithelial cells. Eur Arch Otorhinolaryngol. 2017 Feb;274(2):845-853.

13. Müller C, Sendler M, Hildebrandt JP. Downregulation of aquaporins 1 and 5 in nasal gland by osmotic stress in ducklings, Anas platyrhynchos: implications for the production of hypertonic fluid. J Exp Biol. 2006 Oct;209(Pt 20):4067-76

14. Ma T, Fukuda N, Song Y, Matthay MA, Verkman AS. Lung fluid transport in aquaporin-5 knockout mice. J Clin Invest. 2000 Jan;105(1):93-100.

15. Zhang ZQ, Song YL, Chen ZH, Shen Y, Bai CX. Deletion of aquaporin 5 aggravates acute lung injury induced by Pseudomonas aeruginosa. J Trauma. 2011 Nov;71(5):1305-11. 
16. Yang F, Kawedia JD, Menon AG. Cyclic AMP regulates aquaporin 5 expression at both transcriptional and post-transcriptional levels through a protein kinase A pathway. J Biol Chem. 2003 Aug 22;278(34):32173-80

17. Wang $\mathrm{W}$, Zheng $\mathrm{M}$. Role of cAMP-PKA/CREB pathway in regulation of AQP 5 production in rat nasal epithelium. Rhinology. 2011 Oct;49(4):464-9.

18. Borok Z, Li X, Fernandes VF, Zhou B, Ann DK, Crandall ED. Differential regulation of rat aquaporin-5 promoter/enhancer activities in lung and salivary epithelial cells. J Biol Chem. 2000 Aug 25;275(34):26507-14.

19. Wang $W$, Zheng $M$. Nuclear factor kappa B pathway down-regulates aquaporin 5 in the nasal mucosa of rats with allergic rhinitis. Eur Arch Otorhinolaryngol. 2011 Jan;268(1):73-81. doi: 10.1007/s00405-010-1282-3.

20. Kumari SS, Varadaraj M, Yerramilli VS, Menon AG, Varadaraj K. Spatial expression of aquaporin 5 in mammalian cornea and lens, and regulation of its localization by phosphokinase A. Mol Vis. 2012;18:957-67.

21. Hegyi K, Falus A, Toth S. Elevated CREB activity in embryonic fibroblasts of gene-targeted histamine deficient mice. Inflamm Res. 2007 Aug;56(8):339-44.

22. Canonica GW, Blaiss M. Antihistaminic, anti-inflammatory, and antiallergic properties of the nonsedating second-generation antihistamine desloratadine: a review of the evidence. World Allergy Organ J 2011;4:47-53.

23. Vena GA, Cassano N, Buquicchio R, Ventura MT. Antiinflammatory effects of H1-antihistamines: clinical and immunological relevance. Curr Pharm Des. 2008;14(27):2902-11. Review.

24. Roumestan C, Henriquet C, Gougat C, Michel A, Bichon F, Portet K, Jaffuel D, Mathieu M. Histamine H1-receptor antagonists inhibit nuclear factor-kappaB and activator protein-1 activities via H1-receptor-dependent and -independent mechanisms. Clin Exp Allergy. 2008 Jun;38(6):947-56.

25. Karabasil MR, Hasegawa T, Azlina A, Purwanti N, Purevjav J, Yao C, Akamatsu T, Hosoi K. Trafficking of GFP-AQP5 chimeric proteins conferred with unphosphorylated amino acids at their PKA-target motif ((152)SRRTS) in MDCK-II cells. J Med Invest. 2009 Feb;56(1-2):55-63.

26. Woo J, Chae YK, Jang SJ, Kim MS, Baek JH, Park JC, Trink B, Ratovitski E, Lee T, Park B, Park M, Kang JH, Soria JC, Lee J, Califano J, Sidransky D, Moon C. Membrane trafficking of AQP5 and cAMP dependent phosphorylation in bronchial epithelium. Biochem Biophys Res Commun. 2008 Feb 8;366(2):321-7.

27. Parvin MN, Kurabuchi S, Murdiastuti K, Yao C, Kosugi-Tanaka C, Akamatsu T, Kanamori N, Hosoi K. Subcellular redistribution of AQP5 by vasoactive intestinal polypeptide in the Brunner's gland of the rat duodenum. Am J Physiol Gastrointest Liver Physiol. 2005 Jun;288(6):G1283-91.

28. Kim JH, Park SH, Moon YW, Hwang S, Kim D, Jo SH, Oh SB, Kim JS, Jahng JW, Lee JH, Lee SJ, Choi SY, Park K. Histamine H1 receptor induces cytosolic calcium increase and aquaporin translocation in human salivary gland cells. J Pharmacol Exp Ther. 2009 Aug;330(2):403-12.

29. Li J, Volk A, Zhang J, Cannova J, Dai S, Hao C, Hu C, Sun J, Xu Y, Wei W, Breslin P, Nand S, Chen J, Kini A, Zhu J, Zhang J. Sensitizing leukemia stem cells to NF-KB inhibitor treatment in vivo by inactivation of both TNF and IL-1 signaling. Oncotarget. 2017 Jan 31;8(5):8420-8435.

30. Skowron-zwarg M, Boland S, Caruso N, Coraux C, Marano F, Tournier F. Interleukin-13 interferes with CFTR and AQP5 expression and localization during human airway epithelial cell differentiation. Exp Cell Res. 2007 Jul 15;313(12):2695-702.

31. Aoki Y, Qiu D, Zhao GH, Kao PN. Leukotriene B4 mediates histamine induction of NF-kappaB and IL-8 in humanbronchial epithelial cells. Am J Physiol. 1998 Jun;274(6 Pt 1):L1030-9. 